\title{
Nature of Computation and Communication
}

\author{
Phan Cong Vinh ${ }^{1}$
}

Published online: 22 February 2016

(C) Springer Science+Business Media New York 2016

\section{Foreword}

This special issue, with eight papers invited and extended from selected contributions to The 1 st International Conference on Nature of Computation and Communication (ICTCC 2014) held in Ho Chi Minh City of Vietnam, will serve as a reference material for researchers, scientists, professionals and students in computer science and computer engineering as well as developers and practitioners in computing and networking systems design by providing them with stateof-the-art research findings and future opportunities and trends. These papers include some various aspects of nature of computation and communication reflected in this special issue. In particular, the special issue covers various contributions of nature of computation and communication as follows:

Paper 1 by Phan Cong Vinh considers autonomic computing (AC) characterized by self-* such as self-configuration, selfhealing, self-optimization, self-protection and more which run simultaneously in autonomic systems (ASs). Hence, self-* is a set of self-_'s. Each self-_ in self-* is called self-* action. A way to interpret self-* is to say that self-* actions are running on ASs. In this paper, algebraic objects called monoids are tasked with encoding the self-* action's perspective in all this, i.e. what the self-* action can do, and what happens when different self-* actions are done in succession.

Paper 2 by Nguyen Thanh Tung and Huynh Thi Thanh Binh considers determining an optimal base station location

Phan Cong Vinh

pcvinh@ntt.edu.vn

1 Nguyen Tat Thanh University, Ho chi Minh City, Vietnam

such that the energy consumption is kept lowest, maximizing the network's lifetime and propose a nonlinear programming model for this optimizing problem.

Paper 3 by Waralak V. Siricharoen presents the simple methodologies and methods used to build/adapt/integrate ontologies from the system requirement or by reusing and reengineering other ontologies; moreover a process of merging approach is also considered.

Paper 4 by Huixing Fang, Huibiao Zhu and Jifeng He considers a hybrid modeling language (called HML) for hybrid systems extended with templates to achieve code reuse. For the formal analysis of the corresponding hybrid system models in this modeling language, these models are translated into SMT (satisfiability modulo theories) formulas as the input to an SMT solver dReal which retains the capability of bounded reachability analysis for non-linear hybrid systems. Moreover, dReal can produce data for potential traces of hybrid systems, thus it can be employed to simulate on hybrid systems. In this paper the simulation and reachability analysis are integrated in a prototype tool (open source).

Paper 5 by Donald Elmazi, Shinji Sakamoto, Tetsuya Oda, Elis Kulla, Evjola Spaho and Leonard Barolli proposes and implements two Fuzzy Based Actor Selection Systems (FBASS): FBASS1 and FBASS2. Authors focus on actor selection problem and implement two fuzzy-based system. The systems decide whether the actor will be selected for the required job or not, based on data supplied by sensors and actual actor condition.

Paper 6 by Udsanee Pakdeetrakulwong, Pornpit Wongthongtham, Waralak V. Siricharoen and Naveed Khan proposes an active Software Engineering Ontology through Multi-Agent System (SEOMAS) framework which is intended to provide active support to access software engineering 
domain knowledge and to recommend software project information captured in the SE Ontology. The UML and Agent UML are used to formalize the SEOMAS framework and to model interactions between the various agents. The prototype is developed and evaluated using several scenarios of the online shopping system development as a case study.

Paper 7 by Phan Cong Vinh considers self-* as a foundation for autonomic computing. The notion of autonomic systems (ASs) and self-* serves as a basis on which to build our intuition about category of ASs in general. This paper will specify ASs and self-* and then move on to consider some universal constructions such as products, coproducts, finite limits and colimits of ASs. All of this material is taken as an investigation of the category of ASs.

Paper 8 by Kaiyu Wan, Nhat Nguyen and Vangalur Alagar develops an integrated traffic control system for urban and freeway road network that optimizes the performance of traffic flows in both macro and micro levels, while maintaining safety, liveness, and fairness properties. Towards achieving this goal authors have given efficient adaptive traffic control strategies for optimizing traffic flow and minimizing congestion at urban road intersections, on urban roads, on highway ramps, and on freeways.

We owe our deepest gratitude to Prof. Nguyen Manh Hung-Chairman and Rector of Nguyen Tat Thanh University in Vietnam for his useful support, especially to all the authors for their valuable contribution to this special issue and their great efforts, and also to the referees for ensuring the high quality of the material presented here. All of them are extremely professional and cooperative. We wish to express our thanks to the Editor-in-
Chief, Prof. Imrich Chlamtac, for his important assistance with the process of assembling the special issue.

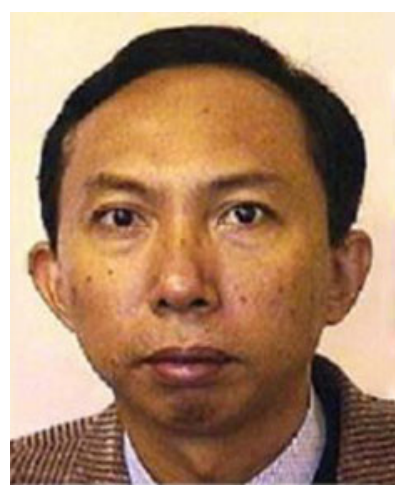

Phan Cong Vinh received a $\mathrm{PhD}$ in computer science from London South Bank University (LSBU) in the United Kingdom. He finished his $\mathrm{PhD}$ dissertation with the title of "Formal Aspects of Dynamic Reconfigurability in Reconfigurable Computing Systems" at LSBU where he was affiliated with the Center for Applied Formal Methods (CAFM) at the Institute for Computing Research (ICR). At present, he is an Associate Professor of Nguyen Tat Thanh University (NTTU) to take on the responsibility of a senior research scientist. He has been author or co-author of many refereed contributions published in prestigious journals, conference proceedings or edited books. He is editor of two books titled, "Autonomic Networking-onChip: Bio-Inspired Specification, Development and Verification" (CRC Press, 2012) and "Formal and Practical Aspects of Autonomic Computing and Networking: Specification, Development and Verification" (IGI Global, 2011). He has served on many conference program committees and has been general or technical (co)chair and (co)organizer of several international conferences such as ICCASA and ICTCC series. His research interests center on all aspects of formal methods, nature of computation and communication, and applied categorical structures in computer science. 